\title{
Internações e óbitos de idosos por influenza no estado de Minas Gerais
}

\author{
Hospitalizations and deaths among the elderly from influenza in \\ the state of Minas Gerais, Brazil
}

\section{Hospitalizaciones y muertes entre los ancianos por influenza en el estado de Minas Gerais, Brasil}

Recebido: 18/06/2013

Aprovado: 30/09/2013
Dayane Aparecida Viana ${ }^{1}$

Lilane Maria Alves Silva ${ }^{2}$

Leiner Resende Rodrigues ${ }^{3}$

O objetivo do presente trabalho foi identificar o número de internações hospitalares e óbitos por Influenza entre os idosos do estado de Minas Gerais no ano de 2010 segundo gênero, período de processamento e faixa etária. Trata-se de um estudo descritivo e analítico, com dados secundários do DATASUS de acordo com os registros de Morbidade Hospitalar do SUS - por local de internação. As análises se procederam em frequências absolutas e relativas e medidas de centralidade e dispersão. As comparações, tanto de internações quanto de óbitos por faixa etária, foram submetidas ao teste $T$-Student, com significância de $p \leq 0,05$. Verificou-se que as mulheres constituíram a maioria das internações hospitalares, e os óbitos mais recorrentes entre os homens, nos meses de junho a setembro, e em indivíduos com 80 anos ou mais. Foi estatisticamente significativa a relação entre óbitos e idosos longevos. Verifica-se a necessidade de mais estudos que avaliem outros fatores associados à Influenza, utilizando outros sistemas de informação.

Descritores: Idoso; Influenza humana; Hospitalização; Morte.

The aim of present study was to identify the number of hospitalizations and deaths from influenza among the elderly in the State of Minas Gerais, Brazil, in 2010 according to gender, processing time and age. This is a descriptive and analytical study, using secondary data from DATASUS according to the records of the Hospital Morbidity SUS - a place of internment. deteccion. The analyzes proceeded in absolute and relative frequencies and measures of centrality and dispersion. Comparisons of both admissions as deaths by age group were subjected to test $T$-Student with a significance of $p \leq 0,05$. It was found that the majority of hospital admissions were found between of women and deaths among men, in the months from June to September and those aged 80 or more. Were significantly statistical relationship between deaths and oldest old. There is a need for more studies that assess other factors associated with influenza, using other information systems.

Descriptors: Aged; Influenza human; Hospitalization, Death.

El objetivo de este estudio fue identificar el número de hospitalizaciones y muertes por la gripe influenza entre las personas mayores en el Estado de Minas Gerais, Brasil, en 2010, según sexo, periodo de procesamiento y edad. Se trata de un estudio descriptivo y analítico, utilizando datos secundarios del DATASUS de acuerdo con los registros de la morbilidad hospitalaria del SUS - por local de hospitalización. El análisis se desarrolló en frecuencias y medidas de centralidad y dispersión absolutas y relativas. Las comparaciones de hospitalización y muertes por grupo de edad fueron sometidas al teste $T$-Student con una significación de $p \leq 0,05$. La mayoría de las hospitalizaciones se encuentrava entre las mujeres y las muertes entre los hombres, en los meses de junio a septiembre y los mayores de 80 años o más. Fueron estadísticamente significativa las muertes en las personas más longevas. Hay necesidad de más estudios que evalúan otros factores asociados con la gripe influenza, con el uso de otros sistemas de información.

Descriptores: Anciano; Gripe humana; Hospitalización; Muerte.

1. Fisioterapeuta. Mestranda em Atenção à Saúde - Universidade Federal do Triângulo Mineiro (UFTM). dayaneviana.fisio@gmail.com

2. Fisioterapeuta. Mestranda em Atenção à Saúde pela UFTM.

3. Enfermeira. Professora Adjunta do Departamento de Enfermagem em Educação e Saúde Comunitária do Curso de Graduação em Enfermagem e da Pós Graduação Strito Senso em Atenção à Saúde da UFTM. 


\section{INTRODUÇÃo}

A s doenças do sistema respiratório são responsáveis pela segunda causa de morbidade hospitalar e pela terceira de mortalidade na população idosa ${ }^{1}$. As infecções respiratórias agudas (IRA) constituem uma síndrome clínica cujos agentes infecciosos mais comuns são os vírus respiratórios ou as bactérias ${ }^{2}$.

A gripe Influenza é uma doença infecciosa aguda do sistema respiratório que acomete as vias aéreas superiores, podendo levar a complicações graves nas vias aéreas inferiores. Os indivíduos acometidos apresentam sintomas tais como: congestão nasal, rinorreia, tosse, rouquidão, febre variável, mialgia e cefaleia. A complicação mais grave e comum são as pneumonias, podendo até levar ao óbito ${ }^{3}$. As doenças crônicas pulmonares podem refletir, de forma indireta, a circulação do vírus Influenza ${ }^{4}$.

O contágio da gripe Influenza pode ocorrer pelo contato direto com secreções respiratórias da pessoa contaminada ao tossir, espirrar e falar, ou por contato indireto ao tocar com as mãos em objetos contaminados e levá-las até a boca, olhos e nariz ${ }^{5}$.

No século passado, ocorreram grandes pandemias como a gripe Espanhola, que em 1918-1919 levou a óbito aproximadamente 50 milhões de pessoas em todo o mundo, a gripe Asiática, em 1957, e a de Hong Kong, em 1968, que dizimaram, cada uma, entre 1-4 milhões de pessoas ${ }^{6,7}$. Em 2009 ocorreu a primeira pandemia de Influenza A do século XXI, pelo vírus Influenza H1N18.

O controle epidemiológico do vírus Influenza no mundo ocorre por meio de uma rede de vigilância da Influenza que está estruturada em 83 países, a qual mobiliza 112 laboratórios. No Brasil, o Sistema de Vigilância Epidemiológica da Influenza foi implantado a partir do ano 2000, e baseia-se em uma estratégia de vigilância sentinela, tendo como objetivos, dentre outros, o monitoramento das cepas virais que circulam nas regiões brasileiras ${ }^{3}$.

Todas as pessoas estão suscetíveis à gripe, porém os idosos, e em especial os institucionalizados e portadores de doenças crônicas, encontram-se entre o grupo de risco que pode sofrer complicações ou mesmo ir a óbito ${ }^{1}$.

Em virtude do crescimento da população idosa e da sua vulnerabilidade, foi implantado pelo Ministério da Saúde em 1999 a vacinação contra a gripe por meio das campanhas anuais, que ocorrem em uma mesma época em todas as regiões, destinadas aos indivíduos com 65 anos ou mais de idade ${ }^{9}$. Em 2000, a vacina passou a ser aplicada em idosos maiores de 60 anos, por meio de um acordo do Ministério da Saúde com o Instituto Butantan ${ }^{10}$.

A campanha, que tem duração de duas a quatro semanas, entre os meses de abril e maio, constitui um dos meios de prevenir a gripe e suas complicações, além de apresentar um impacto indireto na diminuição das internações hospitalares e da morbimortalidade evitável e nos gastos com medicamentos para tratamento e infecções secundárias ${ }^{3}$.

Considerando que a Influenza é uma doença que pode ser prevenida e que os idosos estão entre o grupo populacional de risco, o objetivo deste estudo foi identificar o número de internações hospitalares e óbitos por Influenza entre os idosos do estado de Minas Gerais no ano de 2010.

\section{MÉTODO}

Trata-se de um estudo transversal, descritivo e analítico de dados secundários. Em relação às características éticas da pesquisa, se enfatiza que os dados são anônimos, disponibilizados pelo Ministério da Saúde e, além disso, as informações foram expostas de maneira coletiva e utilizadas somente para atingir o objetivo proposto. Foram revisados os registros de internações hospitalares e mortalidade por Influenza em idosos residentes no estado de Minas Gerais, no ano de 2010, segundo gênero (masculino 
e feminino), faixa etária (60 a 69 anos; 70 a 79 anos e 80 anos ou mais) e mês de processamento (janeiro a dezembro), de acordo com os dados de Morbidade Hospitalar do SUS - por local de internação.

Os dados foram obtidos por meio do Departamento de Informática do Sistema Único de Saúde - DATASUS, disponibilizado pelo Ministério da Saúde via Internet. Foram elaboradas tabelas de acordo com o mês do processamento, gênero e faixa etária, utilizando os recursos do programa Tabnet, também de acesso público. Todos os dados foram selecionados com base na $10^{\underline{a}}$ Classificação Internacional de Doenças (CID10), considerando a Influenza (gripe) como único fator da Lista de Morbidades.

As análises foram realizadas com auxílio do software Statiscal Package for the Social Sciences (SPSS), versão 17.0, com frequências absolutas e relativas e medidas de centralidade e dispersão. As comparações, tanto de internações quanto de óbitos por faixa etária, foram submetidas ao teste T-Student, a fim de verificar a significância entre as três faixas etárias. Foram considerados estatisticamente significativos quando $p \leq 0,05$.

\section{RESULTADOS}

Foram encontrados 748 registros de idosos que foram internados, sendo $43,9 \%$ do sexo masculino e $56,1 \%$ do sexo feminino. Para os óbitos, encontraram-se 70 registros, dos quais $51,4 \%$ eram homens, e $48,6 \%$, mulheres, conforme a Tabela 1 .

Tabela 1 - Internações hospitalares e óbitos de idosos por Influenza de acordo com o gênero. Minas Gerais, 2010.

\begin{tabular}{|c|c|c|c|c|}
\hline \multirow[b]{2}{*}{ Gênero } & \multirow{2}{*}{\multicolumn{2}{|c|}{$\begin{array}{l}\text { Internações } \\
\text { hospitalares }\end{array}$}} & \multicolumn{2}{|c|}{ Óbitos } \\
\hline & & & $\mathrm{N}$ & $\%$ \\
\hline Masculino & 329 & 43,9 & 36 & 51,4 \\
\hline Feminino & 419 & 56,1 & 34 & 48,6 \\
\hline Total & 748 & 100,0 & 70 & 100,0 \\
\hline
\end{tabular}

processamento, verificou-se que as maiores quantidades de internações hospitalares de idosos por Influenza ocorreram em junho, julho e setembro $(9,35 \%, 9,75 \%$ e $11,6 \%$, respectivamente). Não muito diferente dos períodos citados anteriormente, as maiores porcentagens de óbitos foram evidenciadas nos meses de julho $(12,9 \%)$, agosto $(14,9 \%)$ e setembro $(12,9 \%)$, conforme mostra a Tabela 2.

Tabela 2 - Mês de processamento das internações hospitalares e óbitos de idosos por Influenza. Minas Gerais, 2010.

\begin{tabular}{lccrr}
\hline \multirow{2}{*}{ Mês de processamento } & \multicolumn{2}{c}{ Internações hospitalares } & \multicolumn{2}{c}{ Óbitos } \\
Janeiro & $\mathrm{n}$ & $\%$ & $\mathrm{n}$ & $\%$ \\
\cline { 2 - 5 } Fevereiro & 55 & 7,35 & 6 & 8,57 \\
Março & 49 & 6,55 & 5 & 7,14 \\
Abril & 65 & 8,70 & 2 & 2,90 \\
Maio & 48 & 6,41 & 4 & 5,80 \\
Junho & 60 & 8,02 & 7 & 10,00 \\
Julho & 70 & 9,35 & 8 & 11,40 \\
Agosto & 73 & 9,78 & 9 & 12,90 \\
Setembro & 65 & 8,70 & 10 & 14,20 \\
Outubro & 87 & 11,60 & 9 & 12,90 \\
Novembro & 56 & 7,50 & 2 & 2,85 \\
Dezembro & 57 & 7,62 & 5 & 7,14 \\
Total & 63 & 8,42 & 3 & 4,20 \\
\hline
\end{tabular}

Como mostra a Tabela 3, as maiores médias de internações hospitalares $(6,81 \pm 33,5)$ e óbitos $(2,31 \pm 6,49)$ de idosos por Influenza na faixa etária de 80 anos ou mais. Além disso, os dados entre os números de óbitos e a faixa etária foram estatisticamente significativos. 
Tabela 3 - Internações hospitalares e óbitos de idosos por Influenza de acordo com a faixa etária. Minas Gerais, 2010.

\begin{tabular}{|c|c|c|c|}
\hline $\begin{array}{l}\text { Internações Hospitalares } \\
\text { Faixa etária }\end{array}$ & Média & Desvio padrão & $\mathrm{p}$ \\
\hline 60 a 69 anos & 3,66 & 18,10 & 0,475 \\
\hline 70 a 79 anos & 5,45 & 26,80 & 0,872 \\
\hline 80 anos ou mais & 6,81 & 33,50 & 0,602 \\
\hline $\begin{array}{l}\text { Óbitos } \\
\text { Faixa etária }\end{array}$ & & & \\
\hline 60 a 69 anos & 0,69 & 1,99 & 0,0001 * \\
\hline 70 a 79 anos & 1,38 & 3,87 & $0,0001^{*}$ \\
\hline 80 anos ou mais & 2,31 & 6,49 & $0,026^{*}$ \\
\hline
\end{tabular}

*Valores significativos do teste T-Student

\section{DISCUSSÃo}

Alguns autores mostraram a redução do coeficiente de morbidade hospitalar por causas relacionadas à Influenza antes e depois da implantação do programa de vacinação contra Influenza nos idosos ${ }^{11}$. A 15 a Campanha Nacional de Vacinação contra a Influenza revela que a campanha reduz cerca de 50\% das internações hospitalares e óbitos nos indivíduos com 60 anos ou mais e em outros grupos de risco ${ }^{5}$. Pesquisa realizada com dados secundários de 496 municípios gaúchos revelou que aqueles com cobertura vacinal igual ou acima da meta estabelecida de $80 \%$ pelo Ministério da Saúde apresentaram em média 4,5 $\pm 2,93$ mortes por doenças respiratórias em idosos, valor esse inferior ao dos municípios que não atingiram a meta de $80 \%$, que foi de $5,2 \pm 2,68$ mortes $^{12}$.

Investigação realizada sobre morbidade hospitalar mostrou que as doenças respiratórias são a segunda principal causa de internação hospitalar entre idosos nas regiões Sul e Sudeste ${ }^{13}$. No presente estudo, verificou-se que houve maior número de mulheres internadas e maiores porcentagens de óbitos entre os homens. Estudos têm mostrado que a quantidade de pessoas do sexo feminino com 60 anos ou mais é superior à masculina. Além disso, elas são mais dedicadas à saúde, realizam mais consultas médicas e estão sempre atentas ao surgimento de sinais e sintomas de doenças ${ }^{14}$. No entanto, outra pesquisa encontrou resultados divergentes em relação à internação e similares no que se refere aos óbitos encontrados no presente estudo, evidenciando maiores hospitalizações e óbitos no sexo masculino ${ }^{15}$. Porém, isso pode ter ocorrido uma vez que as mulheres aderem mais a campanhas de vacinação e, dessa forma, apresentam fator de proteção ${ }^{16}$.

Uma investigação realizada com 341 idosos residentes em Tubarão-SC elucida que, dos vacinados, $60,6 \%$ eram mulheres ${ }^{17}$. Em Porto Alegre também foram encontrados resultados semelhantes, com maior adesão à vacinação entre idosas $(72,2 \%)^{18}$.

0 pico de casos de Influenza coincide com a estação mais fria do ano, o inverno ${ }^{19}$, dado esse que corrobora os resultados encontrados no presente estudo, o qual evidenciou maiores porcentagens de casos de internações hospitalares e óbitos entre o período de junho a setembro.

Divergente do presente estudo, pesquisa realizada em Fortaleza mostrou que as maiores incidências de internações por doenças respiratórias ocorrem nos meses de abril e maio, o que pode ser explicado pela pouca variação de temperatura dessa região ${ }^{20}$. Outro estudo também realizado na região Nordeste do Brasil, em Aracaju, evidenciou que os maiores índices de internações e taxas de mortalidade por doenças respiratórias nos anos de 1998 a 2007 ocorreram entre os meses de março a junho, mostrando que a campanha de vacinação nessa região deveria ocorrer antecipadamente a esse período ${ }^{21}$.

Estudo sobre a imunização no Brasil verificou que as campanhas vacinais têm sido eficazes na redução dos números de hospitalizações por doenças respiratórias apenas em regiões nas quais o clima é temperado, como no Sul e Sudeste. Já em outras regiões com clima tropical, como o Norte e o Nordeste, os resultados não têm se mostrado positivos ${ }^{22}$. 
$\mathrm{Na}$ presente pesquisa, houve maior número de internações hospitalares e óbitos estatisticamente significativos entre os idosos mais velhos. A gripe é uma infecção comumente relacionada aos indivíduos com maior longevidade, sendo o vírus Influenza um dos seus principais agentes etiológicos, responsável por $75 \%$ das infecções agudas do trato respiratório ${ }^{3}$.

Estudo que investigou a tendência das taxas de mortalidade por doenças respiratórias em idosos nas cinco regiões brasileiras verificou que houve aumento da referida taxa nos indivíduos com 80 anos ou mais $^{23}$. Investigação de séries temporais em Belo Horizonte-MG também corrobora o presente estudo, evidenciando que as taxas de hospitalizações por doenças respiratórias elevam-se com o avanço da idade ${ }^{24}$.

O declínio no sistema fisiológico, juntamente com a dificuldade de manter a homeostase, deixa os idosos longevos mais suscetíveis a doenças ${ }^{25}$.

0 presente estudo utilizou banco de dados secundários que, ainda assim, apresentou dados significativos em relação às maiores médias de óbitos em idosos mais velhos.

\section{CONCLUSÃO}

Verificou-se que as mulheres constituíram a maioria das internações hospitalares, sendo os óbitos mais recorrentes entre os indivíduos do sexo masculino, nos meses de junho a setembro, e naqueles com 80 anos ou mais. Nesse sentido, é preciso intensificar as campanhas de vacinação voltadas para essa população, de maneira que se esclareçam as formas de prevenção da Influenza, mesmo para indivíduos vacinados, e os riscos de se desenvolver outras doenças associadas quando as pessoas não são tratadas, o que pode até levar a óbito. Além disso, é necessário que as campanhas deixem claro que todos os idosos devem procurar os serviços de saúde para aderirem à campanha e também quando notarem qualquer sinal ou sintoma de gripe, principalmente os idosos longevos.

É recomendável mais estudos utilizando outros sistemas de informação, a fim de que sejam avaliados diversos fatores associados às internações hospitalares e óbitos por Influenza em idosos, para que se tenha a possibilidade de redução dessas intercorrências.

\section{REFERÊNCIAS}

1. Lima FSS, Hamman EM. Impacto da vacinação antiinfluenza sobre a morbidade hospitalar e mortalidade por doenças respiratórias na população idosa do Distrito Federal. [dissertação de Mestrado]. Brasília, DF: Universidade de Brasília; 2008. 104p.

2. Cardoso AM. A persistência das infecções respiratórias agudas como problema de saúde pública. Cad. Saúde Pública. 2010; 26(7):1270-1.

3. Ministério da Saúde (Br). Secretaria de Vigilância em Saúde. Departamento de Vigilância Epidemiológica. Coordenação-Geral do Programa Nacional de Imunizações. Influenza: quanto mais prevenção mais proteção. Brasília: Ministério da Saúde; 2010. 21p. (Informe técnico).

4. Donalísio MR, Bergamo PMSF, Lattorre DO. Tendência da mortalidade por doenças respiratórias antes e depois das campanhas de vacinação contra influenza no Estado de São Paulo - 1980 a 2004. Rev. Bras. Epidemiol. 2006; 9(1):32-41.

5. Ministério da Saúde (Br). Sistema Único de SaúdeSUS. Secretarias Municipais e Estaduais de Saúde. Programa Nacional de Imunizações. Campanha Nacional de Vacinação contra a Influenza - ano 2013: quem lembra da vacina se protege da gripe. Brasília: Ministério da Saúde; 2013. 19p. (Informe técnico).

6. Beigel JH, Farrar J, Han AM, Hayden FG, Hyer R, Jong MD et al. Avian influenza A (H5N1) infection in humans. The Writing Committee of the World Health organization (WHO) consultation on human Influenza A/H5. N. Engl. J. Med. 2005; 353(13):1374-85.

7. Taubenberger JK, Morens DM. 1918 Influenza: the mother of all pandemics. Emerg. Infect. Dis. 2006; 12(1):15-22

8. Carneiro M, Trench FJP, Waib LF, Pedro FL, Motta F. Influenza H1N1 2009: revisão da primeira pandemia do século XXI. Rev. AMRIGS. 2010; 54(2):206-13.

9. Nieiro MBP, Alencar GP, Bergamaschi DP. Morbimortalidade por doenças do aparelho respiratório em idosos antes e após a introdução da vacina contra influenza - município de Cubatão, São Paulo, 19992005. São Paulo em Perspect. 2008; 22(2):46-54.

10. Raw I, Higashi HG. Auto-suficiência e inovação na produção de vacinas e saúde pública. Estud. Av. 2008; 22(64):155-70.

11. Dalfenbach LZ, Carmo EH, Duarte EC, Campagna AS, Teles CAS. Morbidade hospitalar por causas relacionadas à influenza em idosos no Brasil, 1992 a 2006. Epidemiol. Serv. Saúde. 2009; 18(1):29-44.

12. Bós AJG, Mirandola AR. Cobertura vacinal está relacionada à menor mortalidade por doenças respiratórias. Cienc. e Saúde Colet. 2013; 18(5):145962. 
13. Ferrer ALM, Marcon SS, Santana RG. Morbidade hospitalar em idosos antes e após vacinação contra influenza no Estado do Paraná. Rev. Latino am. Enferm. 2008; 16(5):31-6.

14. Sales FM, Santos I. Perfil dos idosos hospitalizados e nível de dependência de cuidados de enfermagem: identificação de necessidades. Texto e Contexto Enferm. 2007; 16:495-502.

15. Dalfenbach LZ. Morbidade e mortalidade por influenza no Brasil, no período de 1998 a 2009. In: Ministério da Saúde(Br). Saúde Brasil 2009: uma análise da situação da saúde e da agenda nacional e internacional de prioridades em saúde. Brasília: Ministério da Saúde; 2010. p. 257-78.

16. Heidemann LR, Schuelter-Trevisol F, Machado DFGP, Martins T, Trevisol DJ, Sandin GR. Comparação da morbimortalidade entre idosos vacinados e não vacinados contra Influenza. Rev. Bras. Clin. Med. 2013; 11(1):12-6.

17. Pereira TSS, Freire A, Braga AD, Pereira GW, Blatt CR, Borges AA. Estudo dos efeitos adversos e do efeito protetor da vacina contra influenza em idosos vacinados pela rede pública no município de Tubarão, Estado de Santa Catarina. Rev. Soc. Bras. Med. Trop. 2011; 44(1):48-52.

18. Prass L, Menezes HS, Abegg MP, Gomes MB, Souza WC, Cirino SLMB. Efetividade da vacina contra influenza em idosos em Porto Alegre. Rev AMRIGS. 2010; 54(4):388-92.

19. Harper AS, Fukuda K, Uyeki TM, Cox NJ, Bridges CB. Centers for Disease Control and Prevention (CDC). Advisory Committee on Immunization Practices (ACIP).Prevention and control of Influenza. Recommendations of the Advisory Committee on Immunization Practices (ACIP). MMWR Recomm Rep. 2004; 53(RR-6):1-40. Erratum in: MMWR Recomm Rep. 2004; 53(32):743.
20. Façanha MC. Impacto da vacinação de maiores de 60 anos para influenza sobre as internações e óbitos por doenças respiratórias e circulatórias em Fortaleza - CE/Brasil. J. Bras.. Pneumol. 2005; 31(5):415-20.

21. Gomes AA, Nunes MAP, Oliveira CCC, Lima SO. Doenças respiratórias por influenza e causas associadas em idosos de um município do Nordeste brasileiro. Cad. Saúde Pública. 2013; 29(1):117-22.

22. Cunha SS, Camacho LAB, Santos AC, Dourado 1. Imunização contra influenza no Brasil: racionalidade e desafios. Rev. Saúde Publica. 2005; 39(1):129-36.

23. Campagna AS, Dourado I, Duarte EC, Dalfenbach LZ. Mortalidade por causas relacionadas à influenza em idosos no Brasil, 1992 a 2005. Epidemiol. Serv. Saúde. 2009; 18(3):209-18.

24. Silva MVA, Ferreira MD. Impacto da vacinação contra influenza na morbidade por doenças respiratórias em idosos no município de Belo Horizonte. [Internet]. [citado ago 2013]. Disponível em

http://www.researchgate.net/publication/23707992 2_impacto_da_vacinação_contra_influenza_na_morbid ade_por_doenças_respiratorias_em_idosos_no_municp io_de_belo_horizonte>

25. Tallmann AEC. O cuidado gerontológico de enfermagem e o bem-estar psicológico do idoso longevo. [dissertação de mestrado]. Curitiba: Universidade Federal do Paraná; 2011.109p.

\section{CONTRIBUIÇÕES}

Dayane Aparecida Viana organizou e analisou os dados, elaboração e redação;

Lilane Maria Alves Silva: elaborou a redação final;

Leiner Resende Rodrigues trabalhou na construção e planejamento da pesquisa, na revisão crítica e na redação final. 